\title{
Geometric modular action for disjoint intervals and boundary conformal field theory*
}

\author{
Roberto Longo ${ }^{1}$, Pierre Martinetti ${ }^{2,3}$, Karl-Henning Rehren ${ }^{2,3}$ \\ ${ }^{1}$ Dipartimento di Matematica, \\ Università di Roma 2 "Tor Vergata", 00133 Roma, Italy \\ ${ }^{2}$ Institut für Theoretische Physik, Universität Göttingen, \\ Friedrich-Hund-Platz 1, 37077 Göttingen, Germany \\ ${ }^{3}$ Courant Centre "Higher Order Structures in Mathematics", Universität Göttingen, \\ Bunsenstr. 3-5, 37073 Göttingen, Germany
}

\begin{abstract}
In suitable states, the modular group of local algebras associated with unions of disjoint intervals in chiral conformal quantum field theory acts geometrically. We translate this result into the setting of boundary conformal QFT and interpret it as a relation between temperature and acceleration. We also discuss aspects ("mixing" and "charge splitting") of geometric modular action for unions of disjoint intervals in the vacuum state.
\end{abstract}

Dedicated to John E. Roberts on the occasion of his 70th birthday

\section{Introduction}

Geometric modular action is a most remarkable feature of quantum field theory [2], emerging from the combination of the basic principles: unitarity,

*Supported in part by ERC Advanced Grant 227458 OACFT "Operator Algebras and Conformal Field Theory", and by the EU network "Noncommutative Geometry" MRTNCT-2006-0031962. RL is partially supported by PRIN-MIUR and GNAMPA-INDAM. PM and KHR are supported in part by the German Research Foundation (Deutsche Forschungsgemeinschaft (DFG)) through the Institutional Strategy of the University of Göttingen. 
locality, covariance and positive energy [1. It associates thermal properties with localization [14, 26], and is intimately related to the Unruh effect [30] and Hawking radiation [27]. It allows for a reconstruction of space and time along with their symmetries [6], and for a construction of full-fledged quantum field theories [20, 17] out of purely algebraic data together with a Hilbert space vector (the vacuum).

The modular group [28, Chap. VI, Thm. 1.19] is an intrinsic group of automorphisms of a von Neumann algebra $M$, associated with a cyclic and separating vector $\Phi$, provided by the theory of Tomita and Takesaki [14, 28]. In quantum field theory, $M$ may be the algebra of observables localized in a wedge region $\left\{x \in \mathbb{R}^{4}: x^{1}>\left|x^{0}\right|\right\}$ and $\Phi=\Omega$ the vacuum state. In this situation it follows [1] that the associated modular group is the one-parameter group of Lorentz boosts in the 1-direction, which preserves the wedge, i.e., it has a geometric action on the subalgebras of observables localized in subregions of the wedge.

Geometric modular action was also established for the algebras of observables localized in lightcones or double cones in the vacuum state in conformally invariant QFT [4, 15], and for interval algebras in chiral conformal QFT [16]. It is known, however, that the modular group of the vacuum state is not geometric ("fuzzy") for double cone algebras in massive QFT (see, e.g., [2, 25]), and the same is true for the modular group of wedge algebras or conformal double cone algebras in thermal states [3]. In this contribution, we shall be interested in modular groups for algebras associated with disconnected regions (such as unions of disjoint intervals in chiral conformal QFT).

Our starting point is the observation [18] that in chiral conformal QFT (the precise assumptions will be specified below), for any finite number $n$ of disjoint intervals $I_{i}$ on the circle one can find states (not the vacuum if $n>1$ ) on the algebras $A\left(\bigcup_{i} I_{i}\right)=\bigvee_{i} A\left(I_{i}\right)$ whose modular groups act geometrically inside the intervals.

For $n=2$, let $E=I_{1} \cup I_{2}$ and $E^{\prime}=S^{1} \backslash \bar{E}$ the complement of the closure of $E$. By locality, $A(E) \subset A\left(E^{\prime}\right)^{\prime}$, where the inclusion is in general proper. The larger algebra $A\left(E^{\prime}\right)^{\prime}$ has the physical interpretation as a double cone algebra $B_{+}(O)$ in boundary conformal QFT [21] as will be explained in Sect. 2.2 .

The above state on $A(E)$ can be extended to a state on $B_{+}(O)=A\left(E^{\prime}\right)^{\prime}$ such that the geometric modular action is preserved. We shall compute the geometric flow in the double-done $O$ in Sect. 2. Adopting the interpretation of $\frac{d s}{d \tau}$ as inverse temperature $\beta$ (where $\tau$ is the proper time along an orbit and $s$ the modular group parameter) [10, 24, we compute the relation between temperature and acceleration. There is not a simple proportionality as in 
the case of the Hawking temperature.

In Sect. 3, we shall connect our results with a recent work by Casini and Huerta [8]. In a first quantization approach as in [12], these authors have succeeded to compute the operator resolvent in the formula of [12] for the modular operator. From this, they obtained the modular flow for disjoint intervals and double cones in two dimensions in the theory of free Fermi fields. Unlike [18], they consider the vacuum state. They find a geometric modular action in the massless case (including the chiral case), but this action involves a "mixing" ("modular teleportation" [8]) between the different intervals resp. double cones. Upon descent to gauge-invariant subtheories, the mixing leads to the new phenomenon of "charge splitting" (Sect. 3.3).

Ignoring the mixing, the geometric part of the vacuum modular flow for two intervals in the chiral free Fermi model is the same as the purely geometric modular flow in the non-vacuum product state, provided a "canonical" choice for the latter is made, in the model-independent approach.

We shall make the result of Casini and Huerta (which was obtained by formal manipulations of operator kernels) rigorous by establishing the KMS property of the vacuum state w.r.t. modular action they found. We shall also present a preliminary discussion of the question, to what extent the result may be expected to hold in other than free Fermi theories.

\section{Geometric modular flow for $n$-intervals}

An $n$-interval is the union $E:=\bigcup_{k=1}^{n} I_{k}$ of $n$ open intervals $I_{k} \subset S^{1}(k=$ $1, \ldots, n)$ with mutually disjoint closure. The complement $E^{\prime}=S^{1} \backslash \bar{E}$ is another $n$-interval. If there is an interval $I \subset S^{1}$ such that $E=\left\{z \in S^{1}\right.$ : $\left.z^{n} \in I\right\}$, we write $E=\sqrt[n]{I}$, and call $E$ symmetric. In this case, $E^{\prime}=\sqrt[n]{I^{\prime}}$. Note that every 2-interval is a Möbius transform of a symmetric 2-interval, while the same is not true for $n>2$.

Let $I \rightarrow A(I)$ be a diffeomorphism covariant local chiral net on the circle $S^{1}$. We are interested in the algebras

$$
A(E):=\bigvee_{i=1}^{n} A\left(I_{i}\right) \text { and } \widehat{A}(E):=A\left(E^{\prime}\right)^{\prime},
$$

and their states with geometric modular action. By $\Omega$ we denote the vacuum vector, and by $U$ the projective unitary representation of the diffeomorphism group in the vacuum representation, with generators $L_{n}(n \in \mathbb{Z})$ and central charge $c$. 


\subsection{Product states with geometric modular action}

For $n=1, E$ ist just an interval and $\widehat{A}(I)=A(I)$ (Haag duality).

Proposition 1 (Bisognano-Wichmann property) [16]: The modular group of unitaries for the pair $(A(I), \Omega)$ is given by the one-parameter group of Möbius transformations that fixes the interval $I, \Delta_{A(I), \Omega}^{i t}=U\left(\Lambda_{I}(-2 \pi t)\right)$.

For $I=S_{+}^{1}$ the upper half circle, the generator of the subgroup $U\left(\Lambda_{S_{+}^{1}}(t)\right)$ is the dilation operator $D=i\left(L_{1}-L_{-1}\right)$. It follows that $D$ as well as its Möbius conjugates $D_{I}$ (the generators of the subgroups $U\left(\Lambda_{I}(t)\right)$ ) are "of modular origin":

$$
-2 \pi \cdot D_{I}=\log \Delta_{A(I), \Omega}
$$

Let now

$$
L_{0}^{(n)}=\frac{1}{n} L_{0}+\frac{c}{24} \frac{n^{2}-1}{n}, \quad L_{ \pm 1}^{(n)}=\frac{1}{n} L_{ \pm n},
$$

and $U^{(n)}$ the covering representation of the Möbius group with generators $L_{k}^{(n)}(k=0, \pm 1)$. The unitary one-parameter groups $V(t)=U^{(n)}\left(\Lambda_{I}(-2 \pi t)\right)$ act on the diffeomorphism covariant net by

$$
V(t) A(J) V(t)^{*}=A\left(f_{t}(J)\right) \quad(J \subset \sqrt[n]{I})
$$

where the geometric flow $f_{t}$ is given by (cf. Fig. 1)

$$
f_{t}(z)=\sqrt[n]{\Lambda_{I}(-2 \pi t)\left(z^{n}\right)}
$$

with the branch of $\sqrt[n]{\cdot}$ chosen in the same connected component of $E$ as $z$, i.e., $f_{t}$ is a diffeomorphism of $S^{1}$ which preserves each component of $E$ separately. The same formulae hold also for $J \subset \sqrt[n]{I^{\prime}}$.

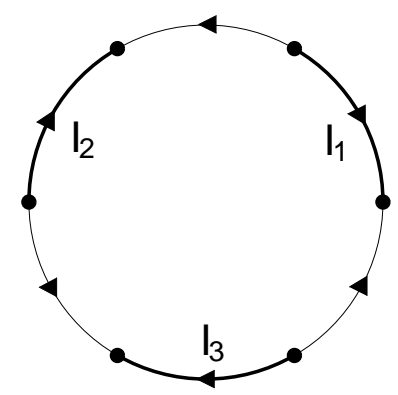

Figure 1: Flow $f_{t}$ in the 3-intervals $E=\sqrt[3]{S_{+}^{1}}=I_{1} \cup I_{2} \cup I_{3}$ and $E^{\prime}=\sqrt[3]{S_{-}^{1}}$.

The question arises whether for $n>1$ the generators $D_{I}^{(n)}$ of $V(t)$ also have "modular origin" as in (2.2). However, unlike with $n=1$, we have the following Lemma and Corollary: 
Lemma: In a unitary positive-energy representation of $\operatorname{sl}(2, \mathbb{R})$ of weight $h>0$, there is no vector such that $D \Phi=0$, where $D=i\left(L_{1}-L_{-1}\right)$.

Proof: An orthonormal basis of the representation is given by the vectors $|n\rangle=\left(n !(2 h)_{n}\right)^{-\frac{1}{2}} L_{-1}^{n}|h\rangle$, where $|h\rangle$ is the lowest weight vector. Solving the eigenvalue equation $L_{1} \Phi=L_{-1} \Phi$ by the ansatz $\Phi=\sum_{n} c_{n}|n\rangle$, produces a recursion for the coefficients $c_{n}$ whose solution is not square-summable.

Corollary: For $n>1$, no cyclic and separating vector $\Phi$ exists in a positive-energy representation of the net $A$ such that the modular Hamiltonian $\log \Delta_{A(E), \Phi}$ would equal $-2 \pi D_{I}^{(n)}$.

Proof: By modular theory, $\log \Delta_{A(E), \Phi} \Phi=0$. But because $L_{0}^{(n)} \geq$ $\frac{c}{24} \frac{n^{2}-1}{n}>0$, the Lemma states that no vector $\Phi$ can be annihilated by $D_{I}^{(n)}$ which is a Möbius conjugate of $D^{(n)}$.

Instead, the appropriate generalization of (2.2) for the modular origin of the generators $D_{I}^{(n)}$ was given in [18], assuming that the net $A$ is completely rational. This means that the split property holds and the $\mu$-index $\mu_{A}$ is finite, and implies the existence of a unique conditional expectation $\varepsilon_{E}$ : $\widehat{A}(E) \rightarrow A(E)$ [19]. In the sequel, $\frac{d \psi}{d \psi^{\prime}}$ is the Connes spatial derivative for a pair of faithful normal states $\psi$ and $\psi^{\prime}$ on a von Neumann algebra $M$ and its commutant $M^{\prime}$, which is a canonical positive operator such that $\left(\frac{d \psi}{d \psi^{\prime}}\right)^{i t}$ implements $\sigma_{t}^{\psi}$ on $M$ and $\left(\frac{d \psi}{d \psi^{\prime}}\right)^{-i t}$ implements $\sigma_{t}^{\psi^{\prime}}$ on $M^{\prime}[9]$.

Proposition 2 [18]: There is a faithful normal state $\varphi_{E}$ on $A(E)(E=$ $\sqrt[n]{I}$ ) and a second faithful normal state $\varphi_{E^{\prime}}$ on $A\left(E^{\prime}\right)$, such that the following hold: The modular automorphism group $\sigma_{t}^{\varphi_{E}}$ is implemented by $V(t), \sigma_{t}^{\varphi_{E^{\prime}}}$ is implemented by $V(-t)$, and

$$
-2 \pi D_{I}^{(n)}=\log \left(\frac{d \widehat{\varphi}_{E}}{d \varphi_{E^{\prime}}}\right)+\frac{n-1}{2} \log \mu_{A} .
$$

Here, $\widehat{\varphi}_{E}=\varphi_{E} \circ \varepsilon_{E}$ extends the state on $A(E)$ to a state on $\widehat{A}(E)$. Moreover, $\frac{d \widehat{\varphi}_{E}}{d \varphi_{E^{\prime}}}=\frac{d \varphi_{E}}{d \widehat{\varphi}_{E^{\prime}}}$.

The state $\varphi_{E}$ on $A(E)$ is given by $\varphi_{E}:=\left(\bigotimes_{k=1}^{n} \varphi_{k}\right) \circ \chi_{E}$ where $\chi_{E}$ : $A(E) \equiv \bigvee_{k=1}^{n} A\left(I_{k}\right) \rightarrow \bigotimes_{k=1}^{n} A\left(I_{k}\right)$ is the natural isomorphism given by the split property $\left(I_{k}\right.$ are the components of $\left.E\right)$, and the states $\varphi_{k}$ on $A\left(I_{k}\right)$ are given by $\varphi_{k}=\omega \circ \operatorname{Ad} U\left(\gamma_{k}\right)$, where $\omega$ is the vacuum state, and $U\left(\gamma_{k}\right)$ implement diffeomorphisms $\gamma_{k}$ that equal $z \mapsto z^{n}$ on $I_{k}$. (By locality, $\varphi_{k}$ do not depend on the behaviour of $\gamma_{k}$ outside $I_{k}$.)

Corollary: Let $\varphi_{E}$ and $\widehat{\varphi}_{E}$ be the states on $A(E)$ and on $\widehat{A}(E)$, resp., as in the Proposition 2. For intervals $J_{k} \subset I_{k}$ (= the components of $E$ ) and 
$F=\bigcup_{k=1}^{n} J_{k}$, we have the geometric modular actions

$$
\begin{gathered}
\sigma_{t}^{\varphi_{E}}\left(A\left(J_{k}\right)\right)=A\left(f_{t}\left(J_{k}\right)\right), \quad \text { hence } \quad \sigma_{t}^{\varphi_{E}}(A(F))=A\left(f_{t}(F)\right), \\
\sigma_{t}^{\widehat{\varphi}_{E}}\left(A\left(J_{k}\right)\right)=A\left(f_{t}\left(J_{k}\right)\right), \quad \text { and } \quad \sigma_{t}^{\widehat{\varphi}_{E}}(\widehat{A}(F))=\widehat{A}\left(f_{t}(F)\right) .
\end{gathered}
$$

Proof: (2.7) is obvious from (2.4). By the defining implementation properties of the Connes spatial derivative, we conclude from (2.6), that $\sigma^{\widehat{\varphi}_{E}}$ is implemented by $V(t)$. This implies (2.8), by the $U^{(n)}$-covariance of the algebras under consideration.

(We include the obvious statement (2.7) for later comparison with the geometric modular flow in [8], for which only the second equality in (2.7) holds while the first is violated.)

For $n=1$, one may just choose $\gamma=\mathrm{id}$, so that both $\varphi_{I}$ and $\varphi_{I^{\prime}}$ are given by the restrictions of the vacuum state, and (2.6) reduces to (2.2).

For $n>1$, the state $\varphi_{E}$ is different from the vacuum state, but it is rotation invariant on $A(E)$ in the sense, that $\varphi_{E} \circ \operatorname{Ad} U\left(\operatorname{rot}_{t}\right)=\varphi_{E}$ on $A\left(J_{k}\right)$ for $\bar{J}_{k} \subset I_{k}$ and $t$ small enough that $\operatorname{rot}_{t}\left(J_{k}\right) \subset I_{k}$. ( $\operatorname{rot}_{t}$ stands for the rotations $z \mapsto e^{i t} z$.) Namely, if $\bar{J} \subset I$ such that $g J \subset I$ for $g$ in a neighborhood $N$ of the identity of the Möbius group, then by construction, $\varphi_{E} \circ \operatorname{Ad} U^{(n)}(g)=\varphi_{E}$ on $A(\sqrt[n]{J})$ for $g \in N$. In particular, the same is true for the rotations rot $_{t}$ with $t$ in a neighborhood of 0 . Since $U^{(n)}\left(\right.$ rot $\left._{t}\right)=$ $U\left(\operatorname{rot}_{t / n}\right) \cdot($ complex phase $)$, the rotation invariance on $A(E)$ follows.

One could actually have chosen any other family of diffeomorphisms $\gamma_{k}$ that map $I_{k}$ onto $I$, resulting in product states $\varphi_{E}^{\left(\gamma_{k}\right)}$ with a different geometric flow on $E$. In that case, the unitary one-parameter group $V(t)$ satisfying the properties of the Proposition 2 is a diffeomorphism conjugate of $U_{I}^{(n)}\left(\Lambda_{I}(-2 \pi t)\right)$. One might expect that our choice of $\varphi_{E}$ is the only one in this class which enjoys the rotation invariance on $A(E)$. Surprisingly, this is not the case:

Let $\varphi_{E}^{\left(\gamma_{k}\right)}$ be a product state on $A(E)$ that is given on $A\left(I_{k}\right)$ by $\omega \circ$ Ad $U\left(\gamma_{k}\right)$, where $\gamma_{k}$ are diffeomorphisms of $S^{1}$ that map $I_{k}$ onto $I$. Then this state is rotation invariant on $A(E)$, by construction, if and only if $\omega \circ \operatorname{Ad} U\left(h_{k}\right)$ are rotation invariant on $A(I)$, where $h_{k}$ are diffeomorphisms of $S^{1}$, defined on $I$ by $h_{k}\left(z^{n}\right)=\gamma_{k}(z)$ for $z \in I_{k}$. In particular, $h_{k}$ map $I$ onto $I$. The condition that $\omega \circ \operatorname{Ad} U(h)$ is rotation invariant on $A(I)$, can be evaluated for the two-point function of the stress-energy tensor in that state. Using the inhomogeneous transformation law under diffeomorphisms $h$, involving the Schwartz derivative $D_{z} h=\frac{h^{\prime \prime \prime}}{h^{\prime}}-\frac{3}{2}\left(\frac{h^{\prime \prime}}{h^{\prime}}\right)^{2}$, the quantity

$$
2 c \cdot\left(\frac{\frac{d h_{t}(z)}{d z} \frac{d h_{t}(w)}{d w}}{\left(h_{t}(z)-h_{t}(w)\right)^{2}}\right)^{2}+\frac{c^{2}}{36} \cdot D_{z} h_{t}(z) \cdot D_{w} h_{t}(w),
$$


where $h_{t}=h \circ \operatorname{rot}_{t}$, must be independent of $t$ for $z, w \in I$ and $t$ in a neighbourhood of zero. Working out the singular parts of the expansion in $w$ around $z$, one finds that $D_{z} h_{t}(z)$ must be independent of $t$ for $z \in I$. This already implies that the second (regular) term is separately invariant, so that, in particular, the invariance condition does not depend on the central charge c. Solving

$$
\partial_{t}\left(D_{z} h_{t}(z)\right)=0 \quad \Leftrightarrow \quad z^{2} \cdot D_{z} h(z)=\text { const. }
$$

when the constant is parametrized as $\frac{1}{2}\left(1-\nu^{2}\right)$, yields

$$
h(z)=\mu\left(z^{\nu}\right)=\frac{A z^{\nu}+B}{C z^{\nu}+D} \quad \text { for } \quad z \in I,
$$

where $\mu$ is a Möbius transformation 1 . The state $\omega \circ \operatorname{Ad} U(h)$ is indeed rotation invariant on $A(I)$ by $h \circ \operatorname{rot}_{t}(z)=\mu \circ \operatorname{rot}_{\nu t}\left(z^{\nu}\right)$ and Möbius invariance of $\omega$.

For each value of $\nu$, requiring $h$ to preserve the endpoints of the interval $I$ fixes the Möbius transformation up to left composition with the one-parameter subgroup $\Lambda_{I}(t)$. Because $\omega$ is invariant under $\Lambda_{I}(t)$, the state $\omega \circ \operatorname{Ad} U(h)$ is uniquely determined by the exponent $\nu$ in (2.11).

One has therefore a one-parameter family of product states, all rotationinvariant on $A(I)$, but with different modular flows on $I$. Going back to the product states on $A(E)$ by composition with $z \mapsto z^{n}$, there is one parameter $\nu_{k}$ for each interval, i.e., for the choice of the states $\omega \circ \operatorname{Ad} U\left(\gamma_{k}\right)$ on $A\left(I_{k}\right)$. The state is invariant also under "large" rotations by $2 \pi / n$, if and only if these parameters are the same for all $k$.

\subsection{Geometric modular action in boundary CFT}

The case $n=2$ is of particular interest in boundary conformal quantum field theory (BCFT) [21]. With every 2-interval $E$ such that $-1 \notin \bar{E}$, one associates a double cone $O_{E}$ in the halfspace $M_{+}=\left\{(t, x) \in \mathbb{R}^{2}: x>0\right\}$ as follows. The boundary $x=0, t \in \mathbb{R}$ is the pre-image of $\dot{S}^{1}:=S^{1} \backslash\{-1\}$ under the Cayley transform $C: \mathbb{R} \ni t \mapsto z=(1+i t) /(1-i t) \in S^{1}$.

\footnotetext{
${ }^{1}$ The sign of the exponent $\nu$ can be reversed by exchanging $A \leftrightarrow B$ and $C \leftrightarrow D$. In order that $h$ takes values in $S^{1}, \nu$ must be either real or imaginary, with corresponding reality conditions on the matrix $\left(\begin{array}{cc}A & B \\ C & D\end{array}\right)$. Requiring $h$ also to preserve the orientation, we find: If $\nu>0$, then $\left(\begin{array}{cc}A & B \\ C & D\end{array}\right) \in S U(1,1)$. If $i \nu>0$, then $\left(\begin{array}{cc}A & B \\ C & D\end{array}\right) \in\left(\begin{array}{cc}i & 1 \\ -i & 1\end{array}\right) \cdot S L(2, \mathbb{R})$, where $\left(\begin{array}{cc}i & 1 \\ -i & 1\end{array}\right)$ is the Cayley transformation $x \mapsto \frac{1+i x}{1-i x}$.
} 
Let $E=I_{-} \cup I_{+} \subset \dot{S}^{1}$ with $I_{-}<I_{+}$in the counter-clockwise order, and $I_{ \pm}^{\mathbb{R}}=C^{-1}\left(I_{ \pm}\right) \subset \mathbb{R}$. Then

$$
O_{E}:=I_{+}^{\mathbb{R}} \times I_{-}^{\mathbb{R}} \equiv\left\{(t, x): t \pm x \in I_{ \pm}^{\mathbb{R}}\right\}
$$

(When there can be no confusion, we shall drop the subscript E.)

Now, the algebras

$$
B_{+}(O):=\widehat{A}(E)
$$

have the re-interpretation as local algebras of BCFT, which extend the subalgebras of chiral observables

$$
A_{+}(O):=A(E) \equiv A\left(I_{-}\right) \vee A\left(I_{+}\right)
$$

Under this re-interpretation, the second statement in (2.8) asserts, that the modular group $\sigma_{t}^{\widehat{\varphi}_{E}}$ acts geometrically inside the associated diamond $O$ :

$$
\sigma_{s}^{\widehat{\varphi}_{E}}\left(B_{+}(Q)\right)=B_{+}\left(f_{s}^{O}(Q)\right),
$$

where the double cone $Q=O_{F} \subset O$ corresponds to a sub-2-interval $F \subset E$, and the flow $f_{s}^{O}$ on $O$ arises from the pair of flows $f_{s}(2.5)$ on $I_{+}$and $I_{-}$, by the said transformations, i.e.,

$$
f_{s}^{O}(t+x, t-x) \equiv\left(u_{s}, v_{s}\right)=\left(C^{-1} \circ f_{s} \circ C(t+x), C^{-1} \circ f_{s} \circ C(t-x)\right) .
$$

For $I_{+}^{\mathbb{R}}=(a, b) \subset \mathbb{R}_{+}$and $I_{-}^{\mathbb{R}}=(-1 / a,-1 / b)$ (corresponding to a symmetric 2-interval $E$ ), we have computed the velocity field

$$
\partial_{s} u_{s}=2 \pi \frac{\left(u_{s}-a\right)\left(a u_{s}+1\right)\left(u_{s}-b\right)\left(b u_{s}+1\right)}{(b-a)(1+a b) \cdot\left(1+u_{s}^{2}\right)}=:-2 \pi V^{O}\left(u_{s}\right)
$$

for $u_{s} \in I_{+}^{\mathbb{R}}$, and the same equation for $v_{s} \in I_{-}^{\mathbb{R}}$.

For $I_{+}^{\mathbb{R}}=\left(a_{1}, b_{1}\right)$ and $I_{-}^{\mathbb{R}}=\left(a_{2}, b_{2}\right)$ corresponding to a non-symmetric 2interval $\tilde{E}$, there is a Möbius transformation $m$ that maps $\tilde{E}$ onto a symmetric interval $E$. Choosing the state $\varphi_{\tilde{E}}:=\varphi_{E} \circ \mathrm{Ad} U(m)$ on $A(\tilde{E})$, the resulting geometric modular flow is given by $\tilde{f}_{s}=m^{-1} \circ f_{s} \circ m$. Going through the same steps, we find

$$
\partial_{s} u_{s}=-2 \pi V^{O}\left(u_{s}\right)=2 \pi \frac{\left(u-a_{1}\right)\left(u-b_{1}\right)\left(u-a_{2}\right)\left(u-b_{2}\right)}{L u^{2}-2 M u+N}
$$

with

$L=b_{1}-a_{1}+b_{2}-a_{2}, \quad M=b_{1} b_{2}-a_{1} a_{2}, \quad N=b_{2} a_{2}\left(b_{1}-a_{1}\right)+b_{1} a_{1}\left(b_{2}-a_{2}\right)$. 
This differential equation is solved by

$$
\log -\frac{\left(u_{s}-a_{1}\right)\left(u_{s}-a_{2}\right)}{\left(u_{s}-b_{1}\right)\left(u_{s}-b_{2}\right)}=-2 \pi s+\text { const. }
$$

The modular orbits for $u=t+x, v=t-x$ are obtained by eliminating $s$ :

$$
\frac{\left(u-a_{1}\right)\left(u-a_{2}\right)}{\left(u-b_{1}\right)\left(u-b_{2}\right)} \cdot \frac{\left(v-b_{1}\right)\left(v-b_{2}\right)}{\left(v-a_{1}\right)\left(v-a_{1}\right)}=\text { const. }
$$

\subsection{General boundary CFT}

Up to this point, we have taken the boundary CFT to be given by $B_{+}(O):=$ $\widehat{A}(E)$, which equals the relative commutant $B_{+}(O)=A(K)^{\prime} \cap A(L)$ by virtue of Haag duality of the local chiral net $A$. Here, $K$ and $L \subset \dot{S}^{1}$ are the open intervals between $I_{+}$and $I_{-}$, and spanned by $I_{+}$and $I_{-}$, respectively, i.e., $L=I_{+} \cup \bar{K} \cup I_{-}$.

The general case of a boundary CFT was studied in [21]. If $A$ is completely rational, every irreducible local boundary CFT net containing $A(E)$ is intermediate between $A(E)$ and a maximal (Haag dual) BCFT net:

$$
A\left(I_{+}\right) \vee A\left(I_{-}\right) \equiv A_{+}(O) \subset B_{+}(O) \subset B_{+}^{\text {dual }}(O) \equiv B(K)^{\prime} \cap B(L),
$$

where $I \mapsto B(I)$ is a conformally covariant, possibly nonlocal net on $\dot{S}^{1}$ (its extension to the circle in general requires a covering), which extends $A$ and is relatively local w.r.t. $A$. If $A$ is completely rational, the local subfactors $A(I) \subset B(I)$ automatically have finite index (not depending on $I \subset \dot{S}^{1}$ ), and there are only finitely many such extensions.

There is then a unique global conditional expectation $\varepsilon$, that maps each $B(I)$ onto $A(I)$. $\varepsilon$ commutes with Möbius transformations and preserves the vacuum state. By relative locality, $\varepsilon$ maps $B(K)^{\prime} \cap B(L)$ into (in general not onto) $A(K)^{\prime} \cap A(L)$, hence

$$
A(E) \equiv A_{+}(O) \subset \varepsilon\left(B_{+}(O)\right) \subset \widehat{A}(E) .
$$

The product state $\widehat{\varphi}_{E}$ on $\widehat{A}(E)$ induces a faithful normal state $\widehat{\varphi}_{E} \circ \varepsilon$ on $B_{+}(O)$.

Proposition 3: In a completely rational, diffeomorphism invariant BCFT, the modular group of the state $\widehat{\varphi}_{E} \circ \varepsilon$ acts geometrically on $B_{+}(Q), Q \subset O$, i.e., $\sigma_{s}^{\widehat{\varphi}_{E} \circ \varepsilon}\left(B_{+}(Q)\right)=B_{+}\left(f_{s}^{O}(Q)\right)$, where $f_{s}^{O}$ is the flow (2.16).

Proof: $B_{+}(O)$ is generated by $A_{+}(O)$ and an isometry $v$ [21] such that every element $b \in B_{+}(O)$ has a unique representation as $b=a v$ with $a \in$ 
$A_{+}(O)$, and $v a=\theta(a) v$ where $\theta$ is a dual canonical endomorphism of $B_{+}(O)$ into $A_{+}(O)$. For a double cone $Q \subset O$, the isometry $v$ may be choosen to belong to $B_{+}(Q)$, in which case $\theta$ is localized in $Q$. We know that the modular group restricts to the modular group of $A_{+}(O)$, which acts geometrically, in particular, it takes $A_{+}(Q)$ to $A_{+}\left(f_{s}^{O}(Q)\right)$. It then follows by the properties of the conditional expectation that $\sigma_{s}^{\widehat{\varphi}_{E}}{ }^{\circ \varepsilon}(v) \equiv v_{s}=u_{s} v$ where $u_{s} \in A(E)$ is a unitary cocycle of intertwiners $u_{s}: \theta \rightarrow \theta_{s} \equiv \sigma_{s}^{\widehat{\varphi}_{E}} \circ \theta \circ \sigma_{s}^{\widehat{\varphi}_{E}-1}$. Since $\sigma_{s}^{\widehat{\varphi}_{E}}$ acts geometrically in $A_{+}(O), \theta_{s}$ is localized in $f_{s}^{O}(Q)$, and $A_{+}\left(f_{s}^{O}(Q)\right) \cdot v_{s}=$ $B_{+}\left(f_{s}^{O}(Q)\right)$. This proves the claim.

Thus, in every BCFT, the modular group of the state $\widehat{\varphi}_{E} \circ \varepsilon$ on $B_{+}\left(O_{E}\right)$ acts geometrically inside the double cone $O_{E}$ by the same flow (2.20), (2.21).

\subsection{Local temperature in boundary conformal QFT}

We shall show that the states $\widehat{\varphi}_{E} \circ \varepsilon$, whose geometric modular action we have just discussed, are manufactured far from thermal equilibrium. We adopt the notion of "local temperature" introduced in [7], where one compares the expectation values of suitable "thermometer observables" $\Phi(x)$ in a given state $\varphi$ with their expectation values in global KMS reference states $\omega_{\beta}$ of inverse temperature $\beta$. If one can represent the expectation values as weighted averages

$$
\varphi(\Phi(x))=\int d \rho_{x}(\beta) \omega_{\beta}(\Phi(x))
$$

(where the thermal functions $\beta \mapsto \omega_{\beta}(\Phi(x)$ ) do not depend on $x$ because KMS states are translation invariant), then one may regard the state $\varphi$ at each point $x$ as a statistical average of thermal equilibrium states. In BCFT, this analysis can be carried out very easily for the product states $\varphi_{E}$ with the energy density $2 T_{00}(t, x)=T(t+x)+T(t-x)$ as thermometer observable. One has $\omega_{\beta}(T(\cdot))=\frac{\pi^{2}}{24} c \beta^{-2}$ in the KMS states, while the inhomogeneous transformation law of $T$ under diffeomorphisms gives $\varphi_{E}(T(y))=-\frac{c}{24 \pi} D_{y} \gamma_{ \pm}(y)=$ $-\frac{c}{4 \pi}\left(1+y^{2}\right)^{-2}$ if $y \in I_{ \pm}^{\mathbb{R}}$ where $\gamma_{ \pm}(y)=C^{-1} \circ\left(z \mapsto z^{2}\right) \circ C(y)=\frac{2 y}{1-y^{2}}$, i.e. negative energy density inside the double cone $O=I_{+}^{\mathbb{R}} \times I_{-}^{\mathbb{R}}$. The product states $\varphi_{E}$ can therefore not be interpreted as local thermal equilibrium states in the sense of [7]. The possibility of locally negative energy density in quantum field theory is well-known, and its relation to the Schwartz derivative in two-dimensional conformal QFT was first discussed in [13]. 


\subsection{Modular temperature in boundary conformal QFT}

The "thermal time hypothesis" [10] provides a very different thermal interpretation of states with geometric modular action. According to this hypothesis, one interprets the norm of the vector $\partial_{s}$ tangent to the modular orbit $x^{\mu}(s)$ as the inverse temperature $\beta_{s}$ of the state as seen by a physical observer with accelerated trajectory $x^{\mu}(s)$. In the vacuum state on the Rindler wedge algebra, this gives precisely the Unruh temperature $\beta_{s}=\frac{d \tau}{d s}=\frac{2 \pi}{\kappa}$ ( $\tau$ is the proper time, and $\kappa$ the acceleration). One may also give a local interpretation, by viewing $\beta_{s}$ as the inverse temperature of the state for an observer at each point whose trajectory is tangent to the unique modular orbit through that point.

For these interpretations to make sense it is important that $\partial_{s}$ is a timelike vector. Indeed, it is easily seen that the flow (2.17), (2.18) gives negative sign for both $\partial_{s} u_{s}$ and $\partial_{s} v_{s}$, because the velocity field $V^{O}$ is positive inside the interval. Hence the tangent vector is past-directed timelike. This conforms with a general result, proven in more than 2 spacetime dimensions:

Proposition 4 [29]: Let $A(O)$ be a local algebra and $U_{t}$ a unitary oneparameter group such that $U_{t} A(Q) U_{t}^{*}=A\left(f_{t} Q\right)$ where $f_{t}$ is an automorphism of $O$ taking double cones in $O$ to double cones. If there is a vector $\Phi$, cyclic and separating for $A(O)$, such that $U_{t} A \Phi$ has an analytic continuation into a strip $-\beta<\operatorname{Im} t<0$, then $-\left.\partial_{t}\left(f_{t} x\right)\right|_{t=0} \in \overline{V_{+}}$. In particular, the flow of a geometric modular action is always past-directed null or timelike.

From (2.18), we get the proper time $(d \tau)^{2}=d u d v$ and hence the inverse temperature $\beta=\frac{d \tau}{d s}$ as a function of the position $x^{\mu}=(t, x)$

$$
\beta(t, x)^{2}=\frac{d u}{d s} \frac{d v}{d s}=4 \pi^{2} \cdot V^{O}(t+x) V^{O}(t-x) .
$$

The temperature diverges on the boundaries of the double cone $\left(V^{O}\left(a_{i}\right)=\right.$ $\left.V^{O}\left(b_{i}\right)=0\right)$, and is positive everywhere in its interior.

For comparison with the ordinary Unruh effect, we also compute the acceleration in the momentarily comoving frame

$$
\kappa=\left(-\frac{\partial^{2} x_{\mu}}{\partial \tau^{2}} \frac{\partial^{2} x^{\mu}}{\partial \tau^{2}}\right)^{\frac{1}{2}}=\frac{\left(d^{2} x / d t^{2}\right)}{\left(1-(d x / d t)^{2}\right)^{3 / 2}}=\frac{u^{\prime \prime} v^{\prime}-u^{\prime} v^{\prime \prime}}{2\left(u^{\prime} v^{\prime}\right)^{3 / 2}},
$$

where the prime stands for $\partial_{s}$, and we have used $\frac{d x}{d t}=\frac{x^{\prime}}{t^{\prime}}=\frac{u^{\prime}-v^{\prime}}{u^{\prime}+v^{\prime}}$ and $\frac{d^{2} x}{d t^{2}}=$ $\frac{(d x / d t)^{\prime}}{t^{\prime}}=4 \frac{u^{\prime \prime} v^{\prime}-u^{\prime} v^{\prime \prime}}{\left(u^{\prime}+v^{\prime}\right)^{3}}$. Thus

$$
\kappa(t, x)=\left.\frac{V^{O^{\prime}}(u)-V^{O^{\prime}}(v)}{2 \sqrt{V^{O}(u) V^{O}(v)}}\right|_{u=t+x, v=t-x}=\frac{V^{O^{\prime}}(t+x)-V^{O^{\prime}}(t-x)}{\pi^{-1} \beta(t, x)}
$$


as a function of the position $(t, x)$. The product

$$
\begin{aligned}
\beta(t, x) \cdot \kappa(t, x) & =\pi\left|\partial_{x}\left(V^{O}(t+x)+V^{O}(t-x)\right)\right| \\
& =\pi\left|\partial_{t}\left(V^{O}(t+x)-V^{O}(t-x)\right)\right|
\end{aligned}
$$

has the maximal value $2 \pi$ (Unruh temperature) near the left and right edges of the double cone, and equals 0 along a timelike curve connecting the past and future tips. This curve is in general not itself a modular orbit.

In general, the modular orbits are not boost trajectories. However, the quantitative departure is very small. As an illustration, we display a true modular orbit, as well as a plot with one coordinate exaggerated by a zoom factor of 100 (Fig. 2).
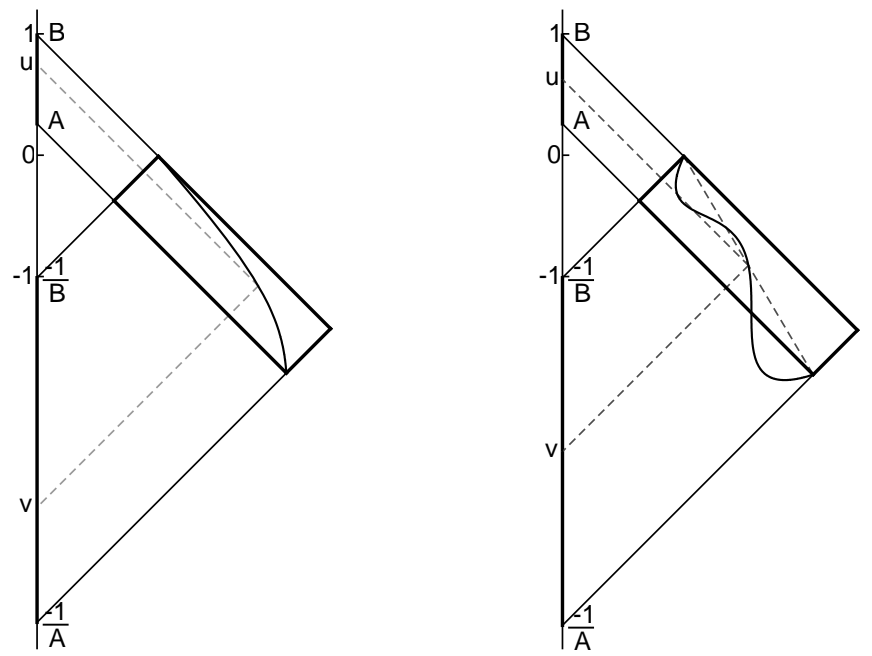

Figure 2: Influence of the boundary. Left: modular orbit of an arbitrary point in the symmetric double cone $O=\left\{(t, x): A \leq t+x \leq B,-\frac{1}{A} \leq t-x \leq-\frac{1}{B}\right\}$. Right: a zoom on the modular orbit $\left(u_{s}, v_{s}\right)$ going through the center of the double cone. The plot represents the curve $\left(\tilde{u}_{s}, v_{s}\right)$ where $\tilde{u}_{s}=f *\left(u_{s}-u_{s}^{\text {diag }}\right)+u_{s}^{\text {diag }}$, with $\left(u_{s}^{\text {diag }}, v_{s}\right)$ the straight line joining the two tips of the double cone (a special vacuum modular orbit in the absence of the boundary), and $f=100$ a zoom factor.

There exists however one distinguished modular orbit with a simple dynamics, namely the boost

$$
u_{s} v_{s}=-1 \quad \forall s \in \mathbb{R}
$$

(in the symmetric case, for simplicity) which is solution of (2.21) for const. = 1. It is the Lorentz boost of a wedge in $M_{+}$, whose edge lies on the boundary $x=0$. The same is true also for non-symmetric intervals, although the formula (2.29) is more involved. 
Along this distinguished orbit the inverse temperature (2.25) simply writes

$$
\beta=2 \pi \frac{\partial_{s} u_{s}}{u_{s}}=2 \pi \frac{d}{d s} \ln u_{s}
$$

One can express the proper time $\tau$ of the observer following the boost as a function of the modular parameter

$$
\tau(s)=\ln u_{s}-\ln u_{0},
$$

hence $\beta(\tau)=2 \pi \frac{V^{O}\left(u_{0} e^{\tau}\right)}{u_{0} e^{\tau}}$. Choosing $u_{0}=1$, one can write the inverse temperature as a function of the proper time in the form

$$
\beta(\tau)=2 \pi \frac{\left(\sinh \left(\tau_{\max }\right)-\sinh (\tau)\right) \cdot\left(\sinh (\tau)-\sinh \left(\tau_{\min }\right)\right)}{\left(\sinh \left(\tau_{\max }\right)-\sinh \left(\tau_{\min }\right)\right) \cdot \cosh (\tau)},
$$

where $\tau_{\min }$ and $\tau_{\max }$ are functions of the coordinates of the double cone. As for double cones in Minkowski space, the temperature is infinite at the tips of the double cone $\left(\tau=\tau_{\min }\right.$ or $\left.=\tau_{\max }\right)$ and reaches its minimum in the middle of the observer's "lifetime". Unfortunately, for generic orbits we have no closed formula for the temperature as a function of the proper time, so as to compare with the "plateau behaviour" (constant temperature for most of the "lifetime") as in [24], that occurs in CFT without boundary for vacuum modular orbits close to the edges of the double cone.

\section{The Casini-Huerta modular flow}

Casini and Huerta recently found [8] that the vacuum modular group for the algebra of a free Fermi field in the union of $n$ disjoint intervals $\left(a_{k}, b_{k}\right) \subset \mathbb{R}$ is given by the formula

$$
\sqrt{\frac{d x_{j}}{d \zeta}} \cdot \sigma_{t}\left(\psi\left(x_{j}\right)\right)=\sum_{k} O_{j k}(t) \sqrt{\frac{d x_{k}(t)}{d \zeta}} \cdot \psi\left(x_{k}(t)\right) .
$$

Here,

$$
e^{\zeta(x)}=-\prod_{k} \frac{x-a_{k}}{x-b_{k}}
$$

defines a uniformization function $\zeta$ that maps each interval $\left(a_{k}, b_{k}\right)$ onto $\mathbb{R}$, and $e^{\zeta} \in \mathbb{R}_{+}$has $n$ pre-images $x_{k}=x_{k}(\zeta)$, one in each interval, i.e., $-\prod_{l} \frac{x_{k}(\zeta)-a_{l}}{x_{k}(\zeta)-b_{l}}=e^{\zeta}$. The geometric modular flow is given by 2

$$
\zeta(t)=\zeta_{0}-2 \pi t
$$

\footnotetext{
${ }^{2}$ In [8], the notation is different: the authors "counter" the flow so that the position of $\sigma_{t}\left(\psi\left(x_{j}(\zeta+2 \pi t)\right)\right)$ remains constant, except for the mixing.
} 
i.e., a separate flow $x_{k}(t)=x_{k}(\zeta-2 \pi t)$ in each interval. The orthogonal matrix $O$ yields a "mixing" of the fields on the different trajectories $x_{i}(t)$, and is determined by the differential equation

$$
\dot{O}(t)=K(t) O(t)
$$

where $K_{j j}(t)=0$ and

$$
K_{j k}(t)=2 \pi \frac{\sqrt{\frac{d x_{j}(t)}{d \zeta}} \sqrt{\frac{d x_{k}(t)}{d \zeta}}}{x_{j}(t)-x_{k}(t)} \quad(j \neq k) .
$$

Remark: The mixing is a "minimal" way to evade an absurd conclusion from Takesaki's Theorem [28, Chap. IX, Thm. 4.2]: Without mixing the modular group would globally preserve the component interval subalgebras. Then the Reeh-Schlieder property of the vacuum vector would imply that the $n$-interval algebra coincides with each of its component interval subalgebras.

Proposition 5: For $\bigcup_{k}\left(a_{k}, b_{k}\right) \subset \mathbb{R}$ the Cayley transform of a symmetric $n$-interval $E=\sqrt[n]{I} \subset S^{1} \backslash\{-1\}$, the geometric part (3.3) of the flow (without mixing) is the same as (2.5).

Proof: We use variables $u_{k}=\frac{1+i a_{k}}{1-i a_{k}}, v_{k}=\frac{1+i b_{k}}{1-i b_{k}}, z=\frac{1+i x}{1-i x}$, and the identity $2 i(x-a)=(1-i x)(1-i a)(z-u)$. Then

$$
e^{\zeta}=-\prod_{k} \frac{x-a_{k}}{x-b_{k}}=\text { const. } \cdot \prod_{k} \frac{z-u_{k}}{z-v_{k}}=\text { const. } \cdot \frac{z^{n}-U}{z^{n}-V}
$$

where $U=u_{k}^{n}, V=v_{k}^{n}$ such that $I=(U, V) \subset S^{1}$. Therefore, the flow (3.3) is equivalent to

$$
\frac{z(t)^{n}-U}{z(t)^{n}-V}=e^{-2 \pi t} \cdot \frac{z^{n}-U}{z^{n}-V}
$$

which in turn is easily seen to be equivalent to (2.5).

Keep in mind, however, that the modular group of the product state in Sect. 2.1 does not "mix" the intervals $\left(a_{k}, b_{k}\right)$.

Since every 2-interval is a Möbius transform of a symmetric 2-interval, the statement of Prop. 5 is also true for general 2-intervals, with the flow (2.20).

\subsection{Verification of the KMS condition}

The authors of [8] have obtained the flow (3.1) using formal manipulations. We shall establish the KMS property of the vacuum state for this flow. Because this property distinguishes the modular group [28, we obtain an independent proof of the claim. 
We take $\bigcup_{k}\left(a_{k}, b_{k}\right) \subset \mathbb{R}$ the Cayley transform of a symmetric $n$-interval $E=\sqrt[n]{I} \subset \dot{S}^{1}$. We first solve the differential equation (3.4) for the mixing.

With angular variables $x=\tan \frac{\xi}{2}$, and $\pi>\xi_{0}>\xi_{1}>\cdots>\xi_{n-1}>-\pi$, the non-diagonal elements of the matrix $K$ can be written as

$$
K_{k l}(t)=2 \pi \cdot \frac{\sqrt{\frac{d x_{k}(t)}{d \xi_{k}(t)}} \sqrt{\frac{d x_{l}(t)}{d \xi_{l}(t)}}}{x_{k}(t)-x_{l}(t)} \sqrt{\frac{d \xi_{k}(t)}{d z}} \sqrt{\frac{d \xi_{l}(t)}{d z}}=2 \pi \cdot \frac{\sqrt{\frac{d \xi_{k}(t)}{d z}} \sqrt{\frac{d \xi_{l}(t)}{d z}}}{2 \sin \frac{\xi_{k}(t)-\xi_{l}(t)}{2}}
$$

for $k \neq l$. For symmetric intervals, $\xi_{k}=\xi_{0}-k \cdot \frac{2 \pi}{n}$ and $\frac{d \xi_{k}}{d z}=\frac{d \xi_{0}}{d z}>0$, hence

$$
K_{k l}(t)=-2 \pi \cdot \frac{\frac{d \xi_{0}(t)}{d z}}{2 \sin \frac{(k-l) \pi}{n}}=\Omega_{k l} \cdot \dot{\xi}_{0}(t), \quad \Omega_{k l}=\frac{1}{2 \sin \frac{(k-l) \pi}{n}} .
$$

With $\Omega=\left(\Omega_{k l}\right)_{k, l=0}^{n-1}$ the constant matrix with vanishing diagonal elements, we obtain the orthogonal mixing matrix

Corollary: The mixing matrix is given by

$$
O(t)=e^{\left(\xi_{0}(t)-\xi_{0}(0)\right) \cdot \Omega} .
$$

Remark: The mixing matrix $O(t)$ always belongs to the same oneparameter subgroup of $S O(n)$, with generator $\Omega$. For $n=2$, this is just

$$
O(t)=\left(\begin{array}{cc}
\cos \theta & -\sin \theta \\
\sin \theta & \cos \theta
\end{array}\right) \quad \text { with } \theta(t)=\frac{1}{2}\left(\xi_{0}(t)-\xi_{0}\right) .
$$

If $E$ is not symmetric, the general formula is

$$
\theta(t)=\arctan \frac{L x_{0}(t)-M}{\sqrt{L N-M^{2}}}-\arctan \frac{L x_{0}(0)-M}{\sqrt{L N-M^{2}}}
$$

with notations as in (2.18) 3

Next, we compute the vacuum expectation values $\left\langle\sigma_{t}\left(\psi\left(x_{i}\right)\right) \sigma_{s}\left(\psi\left(y_{j}\right)\right)\right\rangle$, using (3.1) and $\langle\psi(x) \psi(y)\rangle=\frac{-i}{x-y-i \varepsilon}$. Passing to angular variables $x \mapsto \xi$, $y \mapsto \eta$ by

$$
\frac{\sqrt{d x} \sqrt{d y}}{x-y-i \varepsilon}=\frac{\sqrt{d \xi} \sqrt{d \eta}}{2 \sin \frac{\xi-\eta-i \varepsilon}{2}}
$$

this gives

$$
\begin{aligned}
& \left\langle\sigma_{t}\left(\psi\left(x_{i}\right)\right) \sigma_{s}\left(\psi\left(y_{j}\right)\right)\right\rangle= \\
& \quad=\sum_{k l}\left(e^{\left(\xi_{0}(t)-\xi_{0}\right) \cdot \Omega}\right)_{i k}\left(e^{\left(\eta_{0}(s)-\eta_{0}\right) \cdot \Omega}\right)_{j l} \cdot \frac{-i \sqrt{\frac{d \xi_{k}(t)}{d x_{i}}} \sqrt{\frac{d \eta_{l}(s)}{d y_{j}}}}{2 \sin \left(\frac{\xi_{k}(t)-\eta_{l}(s)-i \varepsilon}{2}\right)} .
\end{aligned}
$$

\footnotetext{
${ }^{3}$ The authors of [8] also compute this angle, but misrepresent it as the arctan of the difference, rather than the difference of the arctan's.
} 
Notice that again $d \xi_{k}, d \eta_{l}$ in the square roots do not depend on $k$ and $l$. To perform the sums over $k$ and $l$, we need a couple of trigonometric identities:

Lemma: For $n \in \mathbb{N}$ and $k=0,1, \ldots n-1$, let $\sin _{k}(\alpha):=\sin \left(\alpha-k \frac{\pi}{n}\right)$. Then (sums and products always extending from 0 to $n-1$ ):

(i) $\prod_{k} \sin _{k}(\alpha)=(-2)^{1-n} \sin (n \alpha)$.

(ii) For $j=0, \ldots, n-1$ one has $\sum_{k: k \neq j} \cot \left((j-k) \frac{\pi}{n}\right)=0$.

(iii) For $j=0, \ldots, n-1$ one has

$$
\sum_{k}\left(e^{2(\alpha-\beta) \Omega}\right)_{j k} \cdot \frac{1}{\sin _{k}(\alpha)}=\frac{\sin (n \beta)}{\sin (n \alpha)} \cdot \frac{1}{\sin _{j}(\beta)} .
$$

Proof: (i) is just another way of writing $\prod_{k}\left(z-\omega_{k}\right)=z^{n}-1$ where $\omega_{k}=e^{i k \frac{2 \pi}{n}}$ are the $n$th roots of unity, and $z=e^{2 i \alpha}$. Dividing (i) by $\sin _{j}(\alpha)$, taking the logarithm, and taking the derivative at $\alpha=0$, yields (ii). For (iii), we have to show that the expression

$$
(-2)^{1-n} \sin (n \alpha) \sum_{k}\left(e^{2 \alpha \Omega}\right)_{j k} \cdot \frac{1}{\sin _{k}(\alpha)}=\left(e^{2 \alpha \Omega}\right)_{j k} \cdot \prod_{l: l \neq k} \sin _{l}(\alpha)
$$

is independent of $\alpha$. Taking the derivative w.r.t. $\alpha$ and inserting (3.9), we have to show that

$$
\sum_{k} \frac{1}{\sin (j-k) \frac{\pi}{n}} \cdot \prod_{l: l \neq k} \sin _{l}(\alpha)+\sum_{k} \cos \left(\alpha-k \frac{\pi}{n}\right) \cdot \prod_{l: l \neq j, k} \sin _{l}(\alpha)=0
$$

Writing $\cos \left(\alpha-k \frac{\pi}{n}\right)=\left(\sin _{k}(\alpha) \cos \left((j-k) \frac{\pi}{n}\right)-\sin _{j}(\alpha)\right) / \sin \left((j-k) \frac{\pi}{n}\right)$, this sufficient condition reduces to the identity (ii).

Using (3.15) with $2 \alpha=\xi_{0}(t)-\eta_{l}(s)$ and $2 \beta=\xi_{0}-\eta_{l}(s)$ in the expression (3.14), and once again with $2 \alpha=\eta_{0}(s)-\xi_{0}$ and $2 \beta=\eta_{0}-\xi_{0}$, we get

$$
\left\langle\sigma_{t}\left(\psi\left(x_{i}\right)\right) \sigma_{s}\left(\psi\left(y_{j}\right)\right)\right\rangle=\frac{\sin \left(n \frac{\xi_{0}-\eta_{0}-i \varepsilon}{2}\right)}{\sin \left(n \frac{\xi_{0}(t)-\eta_{0}(s)-i \varepsilon}{2}\right)} \frac{-i \sqrt{\frac{d \xi_{0}(t)}{d x_{i}}} \sqrt{\frac{d \eta_{0}(s)}{d y_{j}}}}{2 \sin \left(\frac{\xi_{i}-\eta_{j}-i \varepsilon}{2}\right)} .
$$

We exhibit the $t$ - and $s$-dependent terms:

$$
\frac{\sqrt{d \xi_{0}(t)} \sqrt{d \eta_{0}(s)}}{2 \sin \left(\frac{n \xi_{0}(t)-n \eta_{0}(s)-i \varepsilon}{2}\right)}=\frac{\sqrt{d \Xi_{0}(t)} \sqrt{d \mathrm{H}_{0}(s)}}{2 n \sin \left(\frac{\Xi_{0}(t)-\mathrm{H}_{0}(s)-i \varepsilon}{2}\right)}=\frac{1}{n} \frac{\sqrt{d X(t)} \sqrt{d Y(s)}}{X(t)-Y(s)-i \varepsilon} .
$$

The first equality is the invariance of the 2-point function under a Möbius transformation $\mu$ mapping $I$ to $S_{+}^{1}$, such that for $z=e^{i \xi} \in E$ and $w=e^{i \eta} \in E$ 
we get $\mu\left(z^{n}\right)=e^{i \Xi}=\frac{1+i X}{1-i X} \in S_{+}^{1}$ and $\mu\left(w^{n}\right)=e^{i \mathrm{H}}=\frac{1+i Y}{1-i Y} \in S_{+}^{1}$ with $X, Y \in \mathbb{R}_{+}$; the second equality is again (3.13) for the inverse transformation $\Xi \mapsto X, \mathrm{H} \mapsto Y$. By Prop. 5 , the flow on $\mathbb{R}_{+}$is just $X(t)=e^{-2 \pi t} \cdot X$, giving

$$
\left\langle\sigma_{t}\left(\psi\left(x_{i}\right)\right) \sigma_{s}\left(\psi\left(y_{j}\right)\right)\right\rangle=\frac{e^{-\pi(t+s)}}{e^{-2 \pi t} X-e^{-2 \pi s} Y-i \varepsilon} \cdot f\left(x_{i}, y_{j}\right) .
$$

This expression manifestly satisfies the KMS condition in the form

$$
\left\langle\psi(x) \sigma_{-i / 2}(\psi(y))\right\rangle=\left\langle\psi(y) \sigma_{-i / 2}(\psi(x))\right\rangle .
$$

We conclude that the KMS condition holds for the Casini-Huerta flow for symmetric $n$-intervals:

Corollary: For symmetric $n$-intervals $E=\sqrt[n]{I}$, (3.1) is the modular automorphism group of the algebra $A(E)$ with respect to the vacuum state.

Proof: Smearing with test functions of appropriate support, the KMS property holds for bounded generators of the CAR algebra $A(E)$. Because $\psi$ is a free field, the KMS property of the 2-point function in the vacuum extends to the KMS property of the corresponding quasifree (i.e., Fock) state of the CAR algebra.

Remark: It is quite remarkable that by virtue of the mixing, through the identity (ii) of the Lemma, the ratio of the modular vacuum correlation functions

$$
\frac{\left\langle\sigma_{t}^{(n)}\left(\psi\left(x_{i}\right)\right) \sigma_{s}^{(n)}\left(\psi\left(y_{j}\right)\right)\right\rangle}{\left\langle\sigma_{t}^{(1)}(\psi(X)) \sigma_{s}^{(1)}(\psi(Y))\right\rangle}
$$

is independent of the modular parameters $t, s$. Here, in the numerator $\sigma^{(n)}$ is the modular group for a symmetric $n$-interval $\subset \mathbb{R}$, and in the denominator $\sigma^{(1)}$ is the modular group for the 1 -interval $\mathbb{R}_{+}$.

\subsection{Product states for general $n$-intervals}

With hindsight from [8], we can generalize to non-symmetric $n$-intervals the model-independent construction of a product state, as in Sect. 2.1, by replacing the function $z \mapsto z^{n}$ as follows. If $C$ stands for the Cayley transformation $x \mapsto z=\frac{1+i x}{1-i x}$, and $\bigcup_{k}\left(a_{k}, b_{k}\right) \subset \mathbb{R}$ the pre-image of a symmetric $n$-interval $E=\sqrt[n]{I}$, then $U=C\left(a_{k}\right)^{n} \in S^{1}$ and $V=C\left(b_{k}\right)^{n} \in S^{1}$ do not depend on $k$. One computes the uniformization function (3.2) in this case to be given by

$$
e^{\zeta}=C^{-1} \circ \mu \circ\left(z \mapsto z^{n}\right) \circ C(x)
$$


where $\mu: S^{1} \rightarrow S^{1}$ is the Möbius transformation $Z \rightarrow C\left(\frac{(-1)^{n}-V}{(-1)^{n}-U} \cdot \frac{Z-U}{V-Z}\right)$, that takes $I$ to $S_{+}^{1}$. For a general $n$-interval $E=\bigcup I_{k} \subset \dot{S}^{1}$, one may choose $\mu$ an arbitrary Möbius transformation, and replace $z \mapsto z^{n}$ by the function

$$
g(z):=\mu^{-1} \circ C \circ e^{\zeta} \circ C^{-1},
$$

where $\zeta$ is the uniformization function (3.2). Thus, $g$ maps each component $I_{k}$ onto the same interval $I=\mu^{-1}\left(S_{+}^{1}\right)$, i.e., we have $E=g^{-1}(I)$. Repeating the construction of Prop. 2 with factor states $\varphi_{k}=\omega \circ \operatorname{Ad} U\left(\gamma_{k}\right)$, where the diffeomorphisms $\gamma_{k}$ coincide with $g$ on $I_{k}$, one obtains a product state with the geometric modular flow

$$
f_{t}(z)=g^{-1}\left(\Lambda_{I}(-2 \pi t) g(z)\right)
$$

instead of (2.5). By construction, this flow corresponds to $\zeta(t)=\zeta(0)-2 \pi t$ as before, which in turn coincides with the geometric part of the vacuum modular flow (3.1).

\subsection{Lessons from the free Fermi model}

\section{Charge splitting}

It is tempting to ask whether, and in which precise sense, the free Fermi field result extends also to the free Bose case. (The authors of [8] are positive about this, but did not present a proof.) In the chiral situation, the free Bose net $A(I)$ (the current algebra with central charge $c=1$ ) is given by the neutral subalgebras of the complex free Fermi net $F(I)$. Because the vacuum state is invariant under the charge transformation, there is a vacuum-preserving conditional expectation $\varepsilon: F(I) \rightarrow A(I)$, implying that the vacuum modular group of $F(E)$ restricts to the vacuum modular group of $C(E):=\varepsilon(F(E))$. We have

$$
\begin{aligned}
& F(E) \\
& \varepsilon \downarrow \\
& A(E) \subset C(E) \subset \widehat{A}(E),
\end{aligned}
$$

where both inclusions are strict: $C(E)$ contains neutral products of integer charged elements of $F\left(I_{k}\right)$ in different component intervals, which do not belong to $A(E)$, while $\widehat{A}(E)$ contains "charge transporters" [5, 19] for the continuum of superselection sectors of the current algebra with central charge $c=1$, which do not belong to $C(E)$.

Being the restriction of the vacuum modular group of $F(E)$, the action of the vacuum modular group of $C(E)$ can be directly read off. It acts 
geometrically, i.e., takes $C(F)$ to $C\left(f_{t}(F)\right)$ but it does not take $A(F)$ to $A\left(f_{t}(F)\right)$, because the mixing takes a neutral product of two Fermi fields in one component $J_{k}$ of $F$ to a linear combination of neutral products of Fermi fields in different components $f_{t}\left(J_{j}\right)$, belonging to $C\left(f_{t}(F)\right)$ but not to $A\left(f_{t}(F)\right)$. Let us call this feature "charge splitting" (stronger than "mixing").

The inclusion situation (3.26) does not permit to determine the vacuum modular flow of $A(E)$ from that of $C(E)$, because there is no vacuumpreserving conditional expectations $C(E) \rightarrow A(E)$ that would imply that the modular group restricts. (Of course, this would be a contradiction, because we have already seen that the modular group of $F(E)$, and hence that of $C(E)$, does not preserve $A(E)$.) Similarly, we cannot conclude that the vacuum modular flow of $\widehat{A}(E)$ should extend that of $C(E)$, or that of $A(E)$. Prop. 6 below actually shows that this scenario must be excluded.

\section{Application to BCFT}

It is instructive to discuss the consequence of the free Fermi field mixing and the ensuing charge splitting for $C(E)$ under the geometric re-interpretation of boundary CFT, as in Sect. 2.2. For definiteness and simplicity, we consider the case when $A$ is the even subnet of the real free Fermi net, i.e., $A$ is the Virasoro net with $c=\frac{1}{2}$. Unlike the $c=1$ free Bose net, this model is completely rational.

The same considerations as in the previous argument apply also in this case: Again, the inclusions $A(E) \subset C(E):=\varepsilon(F(E)) \equiv F(E)^{\mathbb{Z}_{2}} \subset \widehat{A}(E)$ are strict, the latter because charge transporters for the Ramond sector (weight $h=\frac{1}{16}$ ) do not belong to $C(E)$. The vacuum modular flow for $C(E)$ is induced by that for $F(E)$, but it does not pass to $A(E)$ or $\widehat{A}(E)$.

Let therefore $E \subset \dot{S}^{1}$ be 2-intervals and $O=I_{+}^{\mathbb{R}} \times I_{-}^{\mathbb{R}} \subset M_{+}$the associated double cones. The net

$$
O \mapsto C(O)=F(E)^{\mathbb{Z}_{2}}
$$

is a BCFT net intermediate between the "minimal" net $A_{+}(O)=A(E)$ and the "maximal" (Haag dual) net $B_{+}(O)=\widehat{A}(E)$, see [21]. It is generated by fields $\prod_{i=1}^{n} \psi\left(u_{i}\right) \prod_{j=1}^{m} \psi\left(v_{j}\right)$ with $n+m=$ even, and $u_{i}$ smeared in $I_{+}^{\mathbb{R}}, v_{j}$ smeared in $I_{-}^{\mathbb{R}}$.

The vacuum modular flow of $C(O)$ mixes $f_{t} u_{i}$ with $f_{t} u_{i}^{\prime}$ and $f_{t} v_{j}$ with $f_{t} v_{j}^{\prime}$, where $u \mapsto u^{\prime}$ and $v \mapsto v^{\prime}$ are the bijections of the two intervals onto each other connecting the two pre-images of the uniformization function $\zeta$. Hence, if $\psi(u)^{n} \psi(v)^{m}$ (in schematical notation) belongs to $C(Q)$ for a double

\footnotetext{
${ }^{4}$ Here and below, $F \subset E$ always stands for an $n$-interval $F=\bigcup_{k} J_{k}$ where $J_{k}$ are the components of the pre-image of some interval under the function $\zeta$ (3.2), i.e., in the symmetric case, $F=\sqrt[n]{J}$ with $J \subset I$.
} 
cone $Q \subset O$, the vacuum modular flow takes it to linear combinations of

$$
\psi\left(f_{t} u\right)^{n_{1}} \psi\left(f_{t} u^{\prime}\right)^{n_{2}} \psi\left(f_{t} v\right)^{m_{1}} \psi\left(f_{t} v^{\prime}\right)^{m_{2}}
$$

with $n_{1}+n_{2}=n, m_{1}+m_{2}=m$. Grouping the charged factors to neutral (even) "bi-localized" products, these generators belong to the local algebra of 6 double cones $\bigvee_{\alpha=1}^{6} C\left(f_{t} Q_{\alpha}\right) \subset C\left(f_{t} \widehat{Q}\right)$ around 6 points as indicated in Fig. 3.

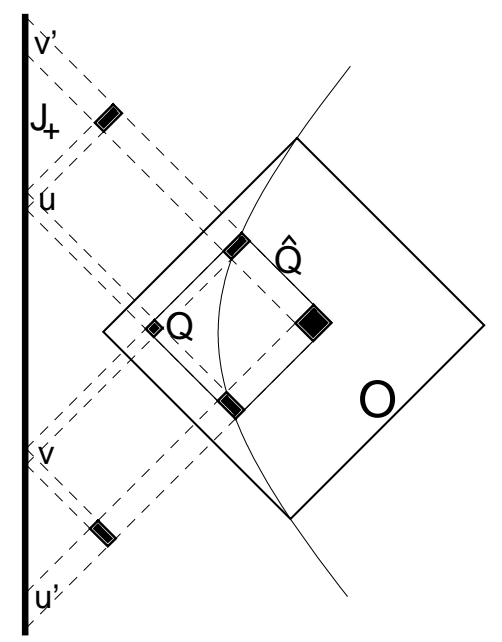

Figure 3: The 6 regions mixed by the vacuum modular flow in boundary CFT. $(u, v)$ is a point in $Q \subset O$. The boost is the distinguished orbit in $O$ as in Sect. 2.5. and defines $u^{\prime}=-\frac{1}{u}$ and $v^{\prime}=-\frac{1}{v}$. If $(u, v)$ lies on the boost, then the points $\left(v, u^{\prime}\right)$ and $\left(v^{\prime}, u\right)$ lie on the boundary. Consequently, if a double cone $Q \subset O$ around $(u, v)$ intersects the distinguished orbit, then four of the 6 associated double cones $Q_{\alpha}$ merge with each other, while the other two touch the boundary and degenerate to left wedges. (The flow $f_{t}$ itself, as in Fig. 2, is suppressed.)

In spite of the fact that two of the 6 double cones $Q_{\alpha}$ lie outside $\widehat{Q}$, the corresponding algebras $C\left(Q_{\alpha}\right)$ are contained in $C(\widehat{Q})$. But their bi-localized generators, such as $\psi(u) \psi\left(v^{\prime}\right)$, cannot be associated with points in $\widehat{Q}$, because on the boundary they are localized in the entire interval $J_{+}$spanned by $u$ and $v^{\prime}$ [22], hence belong to $\bigcap_{J_{-}} C\left(J_{+} \times J_{-}\right) \subset C(\widehat{Q})$. Therefore, in the geometric re-interpretation of boundary CFT, the discrete mixing (charge splitting) on top of the geometric modular action induces a truely "fuzzy" action on BCFT algebras associated with double cones $Q \subset O$ ! The fuzzyness seems, however, not to be described by a pseudo differential operator, as suggested in [26, 25], but rather reflects the nonlocality of an operator product expansion for bilocalized fields. 


\subsection{Preliminaries for a general theory}

Also in the general case of a local chiral net $A$, there is a notion of "charge splitting": Superselection sectors are described by DHR endomorphisms of the local net, which are localized in some interval [11. Intertwiners that change the interval of localization (charge transporters) are observables, i.e., they do not carry a charge themselves, but they may be regarded as operators that annihilate a charge in one interval and create the same charge in another interval. These charge transporters do not belong to $A(E)$, but together with $A(E)$ generate $\widehat{A}(E)$, see the discussion in [19]. Therefore, one may speculate whether the combination of geometric action with charge splitting could be a general feature for the vacuum modular group of suitable $n$-interval

algebras intermediate between $A(E)$ and $\widehat{A}(E)$, i.e., the modular group does not preserve the subalgebras $A(F)$, let alone the algebras of the component intervals $A\left(J_{k}\right)$.

The discussion of the algebras $A(E) \subset C(E) \subset \widehat{A}(E)$ in the preceding subsection shows that there cannot be a simple general answer. Nevertheless, we can derive a few first general results.

Proposition 6: Let $\Phi \in \mathcal{H}$ be a joint cyclic and separating vector for $A(E)$ and $A\left(E^{\prime}\right)$, e.g., the vacuum.

(i) If the modular automorphism group of $(\widehat{A}(E), \Phi)$ globally preserves the subalgebra $A(E)$, then $A(E)=\widehat{A}(E)$.

(ii) If the adjoint action of the modular unitaries $\Delta^{i t}$ for $(A(E), \Phi)$ globally preserves $\widehat{A}(E)$, or, equivalently, $A\left(E^{\prime}\right)$ then $A(E)=\widehat{A}(E)$.

Proof: By assumption, $\Phi$ is also cyclic and separating for $\widehat{A}(E)=A\left(E^{\prime}\right)^{\prime}$ and $\widehat{A}\left(E^{\prime}\right)=A(E)^{\prime}$. Then (i) follows directly by Takesaki's Theorem [28, Chap. IX, Thm. 4.2]. For (ii), note that $\Delta^{i t}$ preserves $A\left(E^{\prime}\right)$ if and only if it preserves $A\left(E^{\prime}\right)^{\prime}=\widehat{A}(E)$; and $\Delta^{-i t}$ implements the modular automorphism group for $\left(A(E)^{\prime}=\widehat{A}\left(E^{\prime}\right), \Phi\right)$. Thus, the statement is equivalent to (i), with $E$ replaced by $E^{\prime}$.

The obvious relevance of (ii) of Prop. 6 is that in the generic case when $\widehat{A}(E)$ is strictly larger than $A(E)$, there can be no vector state satisfying the Reeh-Schlieder property such that $A(E)$ has geometric modular action on $A(E)$ and on $A\left(E^{\prime}\right)$. In particular, the modular unitaries will not belong to the diffeomorphism group, but we may expect that Connes spatial derivatives as in Prop. 2 do.

Recall that we have already seen (in the remark after (3.4)) that mixing necessarily occurs. By (i) of Prop. 6 it is not possible that $\widehat{A}(E)$ has geometric modular action without charge splitting. 


\section{Loose ends}

We have put into relation and contrasted the two facts that

(i) in diffeomorphism covariant conformal quantum field theory there is a construction of states on the von Neumann algebras of local observables associated with disconnected unions of $n$ intervals ( $n$-intervals), such that the modular group acts by diffeomorphisms of the intervals [18], and

(ii) in the theory of free chiral Fermi fields, the modular action of the vacuum state on $n$-interval algebras is given by a combination of a geometric flow with a "mixing" among the intervals [8].

The absence of the mixing in (i) can be ascribed to the choice of "product" states in which quantum correlations across different intervals are suppressed. (In the re-interpretation of 2-interval algebras as double cone algebras in boundary conformal field theory [21], the influence of the boundary was shown to weaken - as expected on physical grounds - in the limit when the double cone is far away from the boundary [22]. Indeed, it can be seen from the formula (3.12) for the mixing angle that in this limit the mixing in (ii) also disappears.) On the other hand, there is some freedom in the choice of product states, which allows to deform the geometric modular flow within each of the intervals. It comes therefore as a certain surprise that the geometric part of the vacuum modular flow in (ii) coincides with the purely geometric flow in the product states in (i), precisely when the latter are chosen in a "canonical" way (involving the simple function $z \mapsto z^{n}$ on the circle, corresponding to $\nu=1$ in (2.11), in the case of symmetric $n$-intervals, and the function $g$ (3.24) in the general case). This means that the relative Connes cocycle between the vacuum state and the "canonical" product state is just the mixing, while for all other product states, it will also involve a geometric component.

Two circles of questions arise:

First, is the geometric part of the vacuum flow specific for the free Fermi model, or is it universal? And if it is universal, what takes the place of the mixing in the general case? Putting aside some technical complications of the proof, the authors of [8] claim a universal behaviour for free fields, while in this paper, we have given first indications how the geometric behaviour should "propagate" to subtheories and to field extensions, also strongly supporting the idea of a universal behaviour. Insight from the theory of superselection sectors suggests that the mixing in the general case should be replaced by a "charge splitting". On the other hand, Takesaki's Theorem poses obstructions against the idea that charge splitting on top of a geometric modular flow could be the general answer (Prop. 6). 
Second, the notion of "canonical" $(\nu=1)$ in the above should be given a physical meaning, related to the absence of a geometric component in the Connes cocycle. In the free Fermi case, the geometric part of the modular Hamiltonian contains the stress-energy tensor $\sim \psi(x) \partial_{x} \psi(x)$, while the mixing part can be expressed in terms of $\psi\left(x_{k}\right) \psi\left(x_{l}\right)$ with $x_{k}$ and $x_{l}$ belonging to different intervals. The absence of derivatives suggests that the Connes cocycle is "more regular in the UV" in the case when the geometric parts coincide, than in the general case. The same should be true for the generalized product state constructed in Sect. 3.2. A precise formulation of this UV regularity is wanted.

Acknowledgments: We thank Jakob Yngvason for bringing to our attention the article of Casini and Huerta [8], and Horacio Casini for discussions about their work. We also thank the Erwin-Schrödinger-Institute (Vienna) for the hospitality at the "Operator Algebras and Conformal Field Theory" program, August-December 2008, where this work has been initiated.

\section{References}

[1] J. Bisognano, E.H. Wichmann: On the duality condition for quantum fields, J. Math. Phys. 17 (1976) 303-321.

[2] H.-J. Borchers: On revolutionizing QFT with modular theory, J. Math. Phys. 41 (2000) 3604-3673.

[3] H.-J. Borchers, J. Yngvason: Modular groups of quantum fields in thermal states, J. Math. Phys. 40 (1999) 601-624.

[4] D. Buchholz: On the structure of local quantum fields with non-trivial interaction, in: Proc. of the Intern. Conf. on Operator Algebras, Ideals, and their Applications in Physics, H. Baumgärtel (ed.), Teubner 1977.

[5] D. Buchholz, G. Mack, I.T. Todorov: The current algebra on the circle as a germ of local field theories, Nucl. Phys. B (Proc. Suppl.) 5B (1988) 20-56.

[6] D. Buchholz, O. Dreyer, M. Florig, S.J. Summers: Geometric modular action and spacetime symmetry groups, Rev. Math. Phys. 12 (2000) 475-560.

[7] D. Buchholz, I. Ojima, H. Roos: Thermodynamic properties of nonequilibrium states in quantum field theory, Annals Phys. 297 (2002) 219-242.

[8] H. Casini, M. Huerta: Reduced density matrix and internal dynamics for multicomponent regions, Class. Quant. Grav. 26 (2009) 185005

[9] A. Connes: On the spatial theory of von Neumann algebras, J. Funct. An. 35 (1980) 153-164.

[10] A. Connes, C. Rovelli: Von Neumann algebra automorphisms and time thermodynamics relation in general covariant quantum theories, Class. Quant. Grav. 11 (1994) 2899-2918. 
[11] S. Doplicher, R. Haag, J.E. Roberts, Local observables and particle statistics, 1+2, Commun. Math. Phys. 23 (1971) 199-230, Commun. Math. Phys. 35 (1974) 49-85.

[12] F. Figliolini, D. Guido: The Tomita operator for the free scalar field, Ann. Inst. H. Poinc.: Phys. Theor. 51 (1989) 419-435.

[13] É.É. Flanagan: Quantum inequalities in two dimensional Minkowski spacetime, Phys. Rev. D 56 (1997) 4922-4926.

[14] R. Haag, N. Hugenholtz, M. Winnink: On the Equilibrium states in quantum statistical mechanics, Commun. Math. Phys. 5 (1967) 215-236.

[15] P. Hislop, R. Longo: Modular structure of the local algebras associated with the free massless scalar field theory, Commun. Math. Phys. 84 (1982) 71-85.

[16] D. Guido, R. Longo: The conformal spin and statistics theorem, Commun. Math. Phys. 181 (1996) 11-35.

[17] D. Guido, R. Longo, H.-W. Wiesbrock: Extensions of conformal nets and superselection structures, Commun. Math. Phys. 192 (1998) 217-244.

[18] Y. Kawahigashi, R. Longo: Noncommutative spectral invariants and black hole entropy, Commun. Math. Phys. 257 (2005) 193-225.

[19] Y. Kawahigashi, R. Longo, M. Müger: Multi-interval subfactors and modularity of representations in conformal field theory, Commun. Math. Phys. 219 (2001) 631-669.

[20] R. Kähler, H.-W. Wiesbrock: Modular theory and the reconstruction of fourdimensional quantum field theories, J. Math. Phys. 42 (2001) 74-86.

[21] R. Longo, K.-H. Rehren: Local fields in boundary conformal QFT, Rev. Math. Phys. 16 (2004) 909-960.

[22] R. Longo, K.-H. Rehren: How to remove the boundary: an operator algebraic procedure, Commun. Math. Phys. 285 (2009) 1165-1182.

[23] R. Longo, F. Xu: Topological sectors and a dichotomy in conformal field theory, Commun. Math. Phys. 251 (2004) 321-364.

[24] P. Martinetti, C. Rovelli: Diamond's temperature: Unruh effect for bounded trajectories and thermal time hypothesis, Class. Quant. Grav. 20 (2003) 49194932.

[25] T. Saffary: On the generator of massive modular groups, Lett. Math. Phys. 77 (2006) 235-248.

[26] B. Schroer, H.-W. Wiesbrock: Modular theory and geometry, Rev. Math. Phys. 12 (2000) 139-158.

[27] G. Sewell: Relativity of temperature and the Hawking effect, Phys. Lett. 79A (1980) 23-24.

[28] M. Takesaki: Theory of Operator Algebras II, Springer Encyclopedia of Mathematical Sciences, Vol. 125 (2003).

[29] S. Trebels: Über die geometrische Wirkung modularer Automorphismen, $\mathrm{PhD}$ Thesis, Göttingen, 1997 (in German, see also [2]).

[30] W.G. Unruh: Notes on black-hole evaporation, Phys. Rev. D 14 (1976) 870892. 